\title{
"FACE MASK CAN MASK PAIN TOO"- ALEXITHYMIA AND ITS IMPLICATIONS FOR PHYSIOTHERAPISTS DURING THE COVID-19 PANDEMIC
}

\author{
Dr. Harish S Krishna 1\& Cyanna Joseph D'souza ${ }^{2}$ \\ 1. PhD, HOD Sharp Insight Rehabilitation Centre, Mangalore, India. \\ Email: sharpinsightimaging@gmail.com \\ 2. MPT, Laxmi memorial College of Physiotherapy, Mangalore, India \\ Email: cyannadsouza11@gmail.com
}

\begin{abstract}
Pain is an unavoidable strand of joint mobilization when dealing with a stiff joint. During this pandemic, patients who undergo this procedure using face mask, risk masking their facial expressions too. Facial grimaces are the ones that a physiotherapist looks out for when gliding a sore joint to get an input on the force they need to apply to the joint. This observational research was carried out on sixteen patients with upper limb pain and stiffness due to varied reasons. The pain endured during mobilization of painful/stiff joints was measured using the Visual Analogue Scale (VAS). The score was calculated without mask on five consecutive days, and then with mask for another five days. Comparison of the mean VAS scores was done using the Independent sample test. The study showed that the patients experienced more pain during joint mobilization when they were masked. There was an average difference of $15 \mathrm{~mm}$ between the mean VAS scoring conditions $(p<0.05)$. Therefore, it was concluded that when patients were wearing face masks, there was a greater sense of pain during joint mobilization than without a mask.
\end{abstract}

Key words: alexithymia, covid-19, face mask, joint mobilization, pain, pandemic 


\section{Introduction}

As we all face unprecedented and unforeseeable challenges due to the ongoing COVID-19 or "Corona- Virus" pandemic, more and more clinical concepts and applications are emerging. In addition to the possibilities and applications of new and current rehabilitation technology, such as telemedicine or telerehabilitation, the safety of patients and therapists must be considered, especially when a gradual return to face-to-face therapy occurs. [1] The World Health Organization (WHO-2020) continues to adapt its recommendation on when and how to use various types of masks during the ongoing pandemic. We can only imagine what will happen to our nonverbal communication skills, given that most of our emotions and feelings are communicated through the face.

May our facial mimicry be influenced or altered by wearing surgical masks, which can lead to a kind of alexithymia, "surgical mask alexithymia" based on the Russell Model? [2] Advanced manual treatment, such as joint mobilization, may not necessarily achieve its proposed objectives when emotion detection and speech are blurred. Clinicians should be mindful that facial deprivation during the COVID-19 pandemic may have a profound effect on the pain and quality of life for an indefinite period of time in conjunction with the enforced social restrictions.

Facial expressions are a source of evidence of pain in actions. It is often face gestures that offer signs of physical discomfort. This face-to-face experience, with both the patient and the treating physiotherapist, while wearing a mask is greatly reduced.[3] According to Maitland, a therapist while mobilizing a painful or stiff joint, should be observant, alert and receptive. ${ }^{[4]}$ Nonverbal communication can give the therapist feedback about the amount of pain a patient is experiencing. This is to prevent occupational hazards related to over pressure, especially in cases of ligament tear, over strained tissues and fragile joints. The therapist is known to get a feedback regarding the amount of pain the patient go through by a nonverbal communication. [5]

The need to study this subject emerged when patients, who were undergoing mobilization before the nationwide lockdown, claimed to have experienced increased pain during the treatment once pandemic began. Hence, it was hypothesized that wearing a mask could be a potential factor contributing to this rise in pain levels. To the authors' knowledge, this research is a first of its kind and will add to the growing evidence of improved patient management during these challenging times.

\section{Materials and Methods}

This descriptive observational study recruited a total of 16 patients between ages 35 to 60 years who were suffering from upper limb stiffness due to varied reasons viz., reflex sympathetic dystrophy (RSD), spondyloarthropathy and rheumatoid arthritis. The study was conducted between February 2020 to April 
2020. A written informed consent was obtained from each participant.

Procedure: Demographic characteristics such as age and BMI were recorded at baseline. All patients were undergoing peripheral joint mobilization (Maitland Grades I-IV) in addition to pain relieving modalities. The treatment was provided by a single therapist. The scores of The Visual Analogue scale (VAS) were recorded without mask (before pandemic) over five consecutive days. During the pandemic the same patients received the same treatment, and five consecutive days of VAS scores were registered with mask.

Outcome Measure: VAS is a unidimensional indicator of the severity of pain that has been extensively used in several adult populations. it has proven to have excellent test-retest and intraclass reliability.[6,7] The VAS is a straight $100 \mathrm{~mm}$ horizontal whose ends are defined as the extreme limits of pain intensity orientated from the left (best) to the right (worst). The patient marks on the line the point that they believe reflects their understanding of their current situation. The VAS score is determined by measuring in millimetres from the left end of the line to the point marked by the patient. 8 The scores can be interpreted as no pain $(0-4 \mathrm{~mm})$, mild pain (5-44 $\mathrm{mm})$, moderate pain (45-74 mm), and severe pain $(75-100 \mathrm{~mm}){ }^{9}$

Data Analysis: Statistical package SPSS (IBM SPSS Statistics for Windows, ver. 21.0. Armonk, NY: IBM Corp.) was used to analyze the data. Descriptive statistics were used to calculate the mean and Standard Deviation (SD) from Dayl to Day5 with and without mask respectively. The paired sample t-test was used to compare the means of the two testing conditions. $p$ value less than 0.05 was considered significant.

\section{Results and Discussion}

All 16 participants completed the study. The mean age and BMl of participants were $51.61 \pm 1.78$ years and $25.04 \pm 1.43$ $\mathrm{kg} / \mathrm{m}^{2}$ respectively. The mean VAS scores (in $\mathrm{mm}$ ) determined from day 1 to day 5 with and without mask are displayed in Table 1. A statistically significant increase in pain intensity was noted when the mean VAS scores (in $\mathrm{mm}$ ) were compared in the two test conditions $(p<0.05)$. The mean difference in the VAS scores is shown in Table 2. Figure 1 indicates the mean VAS scores (in $\mathrm{mm}$ ) calculated from day 1 to day 5 with and without the mask.

Pain expressed by the face is one of the chief nonverbal interactions between the patient and therapist. The Facial Action Coding Scheme is widely used for the characterization of facial gestures in the medical field.[10] Six facial action categories were identified by Craig and Patrick when a person is in pain. [1] Several experiments on nociceptive stimulation and joint range of motion have revealed similar facial expressions when pain is elicited.[12-14] Out of these expressions, it is noteworthy that only one is expressed through the eyes and rest by the mouth, which is obscured whilst wearing a mask. 
Even the eye behavior is often difficult to note when the patient wears a spectacle.

The study found that the pain discomfort could not be spontaneously expressed by patients wearing a mask while undergoing joint mobilization. For a therapist, this serves as a significant barrier to modulating the force they exert on a joint. Feedback on the amount of force they use when mobilizing a joint comes mainly from the form of end range of a joint the therapist experiences, and then from the nonverbal contact they get from the patient's face. This is critical when exercising an extremely painful joint such as in RSD cases. From an evolutionary point of view, the lower part of the face is our first reflex to evaluate a person whether he/ she is in disgust, rage or happiness. ${ }^{15,16}$

Patients with chronic pain due to medical conditions like RSD, stroke, parkinsonism are less precise in expressing their emotions. With added occlusion of the mouth it can contribute to emotional blindness, called alexithymia. ${ }^{17}$ For a specific stimulus, partial face coverage can mask our true emotions. This phenomenon is termed as facial blending. ${ }^{16}$ The authors therefore recommend therapists to keep in mind this scenario in order to prevent complications. The results of the study can be confirmed in a larger sample size and more rigorous research in this area

\begin{tabular}{|l|l|c|c|}
\hline Table 1. Demographic characteristics of VAS in the two testing conditions \\
\hline \multirow{2}{*}{ Day 1 } & Sample Size & $\begin{array}{c}\text { Mean } \pm \text { Standard Deviation } \\
\text { (in mm) }\end{array}$ \\
\cline { 2 - 4 } & With mask & 16 & $75.62 \pm 0.892$ \\
\hline \multirow{2}{*}{ Way2 } & Without mask & 16 & $60.00 \pm 1.366$ \\
\cline { 2 - 4 } & Without mask & 16 & $70.63 \pm 1.123$ \\
\hline \multirow{2}{*}{ Day 3 } & With mask & 16 & $57.50 \pm 1.290$ \\
\cline { 2 - 4 } & Without mask & 16 & $76.20 \pm 1.024$ \\
\hline \multirow{2}{*}{ Day 4 } & With mask & 16 & $58.75 \pm 1.310$ \\
\cline { 2 - 4 } & Without mask & 16 & $73.12 \pm 1.195$ \\
\hline \multirow{2}{*}{ Day 5 } & With mask & 16 & $59.36 \pm 1.389$ \\
\cline { 2 - 4 } & Without mask & 16 & $75.00 \pm 1.095$ \\
\hline
\end{tabular}




\begin{tabular}{|l|c|c|c|}
\hline \multicolumn{4}{|c|}{ Table 2: Mean differences in VAS Scores between the two testing conditions } \\
\hline & $\begin{array}{c}\text { Mean Difference } \pm \\
\text { Standard Error } \\
\text { (in mm) }\end{array}$ & t value & p value \\
\hline Day 1 & $15.62 \pm 0.480$ & 3.830 & .001 \\
\hline Day 2 & $13.13 \pm 0.428$ & 3.068 & .005 \\
\hline Day 3 & $17.45 \pm 0.416$ & 4.208 & .000 \\
\hline Day 4 & $13.76 \pm 0.458$ & 3.001 & .005 \\
\hline Day 5 & $17.50 \pm 0.392$ & 4.456 & .000 \\
\hline
\end{tabular}

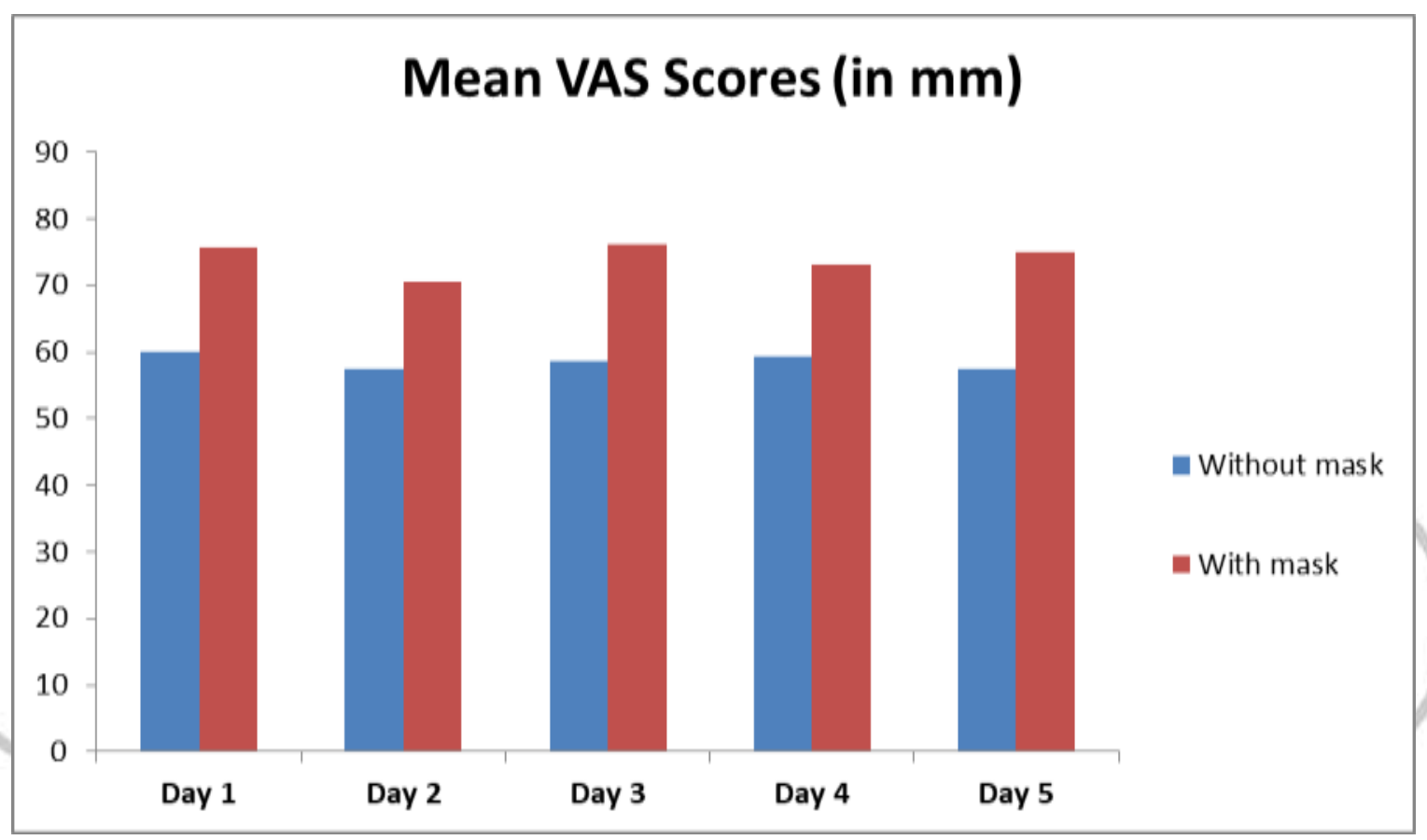

Figure 1: Comparison in the mean VAS scores with and without mask

\section{Conclusion}

Patients who undergo joint mobilization experience greater pain with a mask than without a mask. Due to the current viral disease scenario, wearing a mask is rather an obligation than choice, and clearly an unavoidable factor. A healthy solution is to warn patients about this important miscommunication and ask them to inform them about their pain status verbally. Another alternative to mask is a face shield. On the other side, a call bell may be given for those patients who are unable to communicate clearly.

\section{Declaration of conflicting interests}


The authors declared no conflicts of interest with respect to the authorship and/or publication of this article.

\section{Funding}

The authors received no financial support for the research and/or authorship of this article.

\section{Acknowledgement}

We would like to express our sincere gratitude to the study participants.

\section{References}

1. Eccleston C, Blyth FM, Dear BF, et al. Managing patients with chronic pain during the COVID-19 outbreak: considerations for the rapid introduction of remotely supported (eHealth) pain management services. Pain. 2020; 161: 889-93.

2. Russell JA. Core affect and the psychological construction of emotion. Psychol Rev. 2003; 110: 14572.

3. Kotsia I, Buciu I \& Pitas I. An analysis of facial expression recognition under partial facial image occlusion. Image and Vision Computing. 2008; 26: 1052-67.

4. Maitland GD, 1924-2010. Physical Therapy. 2010; 90(3): 326.

5. Tragord BS, Gill NW, Silvernail JL, et al. Joint mobilization forces and therapist reliability in subjects with knee osteoarthritis. J Man Manip Ther. 2013; 21: 196-206.

6. Alghadir AH, Anwer S, labal A, et al. Test-retest reliability, validity, and minimum detectable change of visual analog, numerical rating, and verbal rating scales for measurement of osteoarthritic knee pain. J Pain Res. 2018; 11:851-56.

7. Hjermstad MJ, Fayers PM, Haugen DF, et al. Studies comparing numerical rating scales, verbal rating scales, and visual analogue scales for assessment of pain intensity in adults: a systematic literature review. J Pain Symptom Manage. 2011; 41: 1073-93.

8. Gould D. Visual Analogue Scale (VAS). Journal of Clinical Nursing. 2001; 10: 697-706.

9. Aun C, Lam YM, Collett B. Evaluation of the use of visual analogue scale in Chinese patients. Pain. 1986; 25: 21521.

10. LeResche L. Facial expression in pain: A survey of candid photographs. J Nonverbal Behav. 1982; 7: 45-56.

11. Craig KD, Patrick CJ. Facial expression during induced pain. J Pers Soc Psychol. 1985; 48: 1080-91.

12. Prkachin KM. The consistency of facial expressions of pain: A comparison across modalities. Pain. 1992; 51: 297-306.

13. Prkachin KM, Solomon PE. The structure, reliability and validity of pain expression: Evidence from patients with shoulder pain. Pain. 2008; 139: 267-74.

14. Grunau RV, Craig KD. Pain expression in neonates: Facial action and cry. Pain. 1987; 28: 395-410. 
15. Ross ED, Prodan $\mathrm{Cl}$, Monnot $\mathrm{M}$. Human Facial Expressions are organized functionally across the upper-lower facial axis.

Neuroscientist. 2007; 13: 433-46.

16. Wegrzyn $M$, Vogt $M$, Kireclioglu $B$, et al. Mapping the emotional faceHow individual face parts contribute to successful emotion recognition. PloS one. 2010; 12: e0177239.
17. Taylor G J, Bagby RM. New trends in alexithymia research. Psychotherapy and psychosomatics. 2004; 73: 68-77.

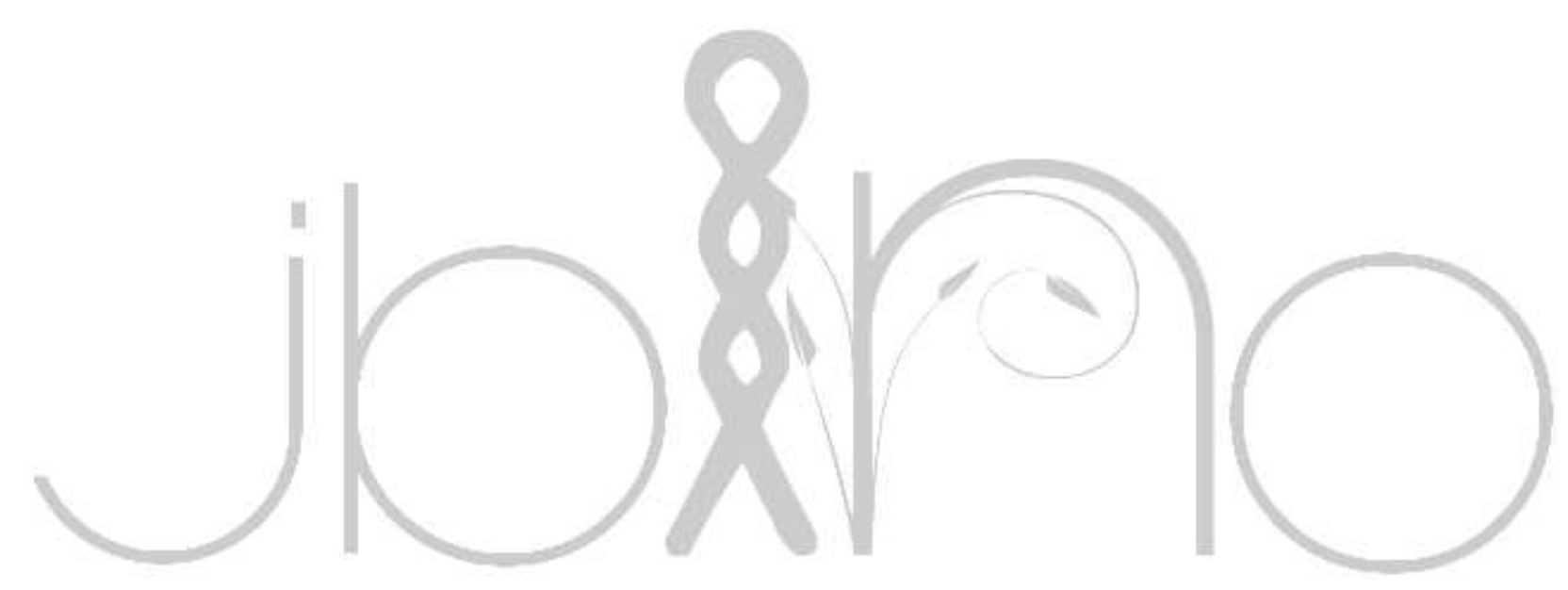

